\title{
Gradual Immobilization Processes of Molecules during Transitions from Solute to Solid States
}

\author{
Guoliang Dai, ${ }^{\dagger, *}$ Gen Sazaki, ${ }^{*}, \$, \#$ Takuro Matsui, ${ }^{\star \$, \|}$ Katsuo Tsukamoto, ${ }^{\perp}$ \\ Kazuo Nakajima, ${ }^{\S, \nabla}$ Qi Kang, ${ }^{\dagger}$ and Wenrui $\mathrm{Hu}^{\dagger}$ \\ ${ }^{\dagger}$ National Microgravity Laboratory, Institute of Mechanics, Chinese Academy of Sciences, \\ Beijing 100190, China, ${ }^{*}$ Center for Interdisciplinary Research, Tohoku University, Sendai 980-8577, \\ Japan, ${ }^{\S}$ Institute for Materials Research, Tohoku University, Sendai 980-8577, Japan, "New Technology \\ Laboratory, Merck Ltd. Japan, Kanagawa 243-0303, Japan, and ${ }^{\perp}$ Graduate School of Science, \\ Tohoku University, Sendai 980-8577, Japan. ${ }^{\#}$ Present address: Institute of Low Temperature Science, \\ Hokkaido University, N19-W8 Kita-ku, Sapporo 060-0819, Japan. ${ }^{\nabla}$ Present address: Graduate School \\ of Energy Science, Kyoto University, Yoshida-honmachi, Sakyo-ku, Kyoto 606-8501, Japan.
}

Received July 1, 2010; Revised Manuscript Received October 24, 2010

\begin{abstract}
The adsorption/desorption behavior of mobile solute molecules at a solution-crystal interface has been explored using crystals of model protein hen egg-white lysozyme (HEWL) and fluorescent-labeled HEWL (F-HEWL) molecules. We have tracked the transient processes occurring during adsorption/desorption of identical F-HEWL molecules on a tetragonal HEWL crystal surface by single-molecule visualization using a total internal reflection fluorescent microscope and pulsed discontinuous laser illumination. We found an induction period ( $\sim 70 \mathrm{~min})$ after which the number density of F-HEWL molecules adsorbed mainly on steps increased linearly with the adsorption time. We show direct evidence that the residence time of molecules on the crystal surface gradually increases during the transition process from a solute species to the crystal after successive multistep processes.
\end{abstract}

\section{Introduction}

In solution crystallization, gradual immobilization of solute molecules at a crystal surface during adsorption/desorption processes happens. Although some studies have attempted to clarify adsorption/desorption processes noninvasively by a single-molecule visualization technique, ${ }^{1-3}$ however, no one has yet succeeded in imaging the transient processes occurring during the discontinuous change of the molecules' state that accompanies the growth of a crystal from a solution. Thus, the transient processes are still mysterious. Our poor understanding of the transient processes is mainly due to the absence of studies attempting to track this process by noninvasive, molecularlevel observation techniques. Scanning probe microscopy, a popular technique for in situ imaging of a solid surface on a molecular level, is not suitable because of significant disturbance of mobile molecules by the tip during a scan.

To our knowledge, the most promising observation technique is single-molecule visualization by total internal reflection fluorescent microscopy,${ }^{4-8}$ by which one can noninvasively track the movement of individual fluorescent molecules at a submicrometer size scale. By this method, diffusion and adsorption processes of different kinds of individual molecules, such as protein ${ }^{2,9}$ and DNA, ${ }^{10}$ were observed at interfaces between solutions and various sorts of solids. ${ }^{2,3,9-13}$ However, using single-molecule visualization to observe the transient processes of crystal growth from a solution has two requirements. First, one has to use a target solute molecule modified with the fluorescent label that is necessary for visualization, while maintaining a close resemblance to those molecules that constitute the solid. Second, one must track identical molecules with high time resolution throughout the transient process. To achieve the first requirement, the target molecule

*To whom correspondence should be addressed. Telephone and Fax: +81-11-706-6880. E-mail: sazaki@lowtem.hokudai.ac.jp. has to be significantly larger than the fluorescent label so that the label has no significant effects on the adsorption process of the target molecule. As such a model system, we chose fluorescent-labeled HEWL molecules and HEWL crystals. ${ }^{14}$ The particular model protein solute utilized for this study was hen egg-white lysozyme (HEWL) labeled with the fluorescent dye tetramethylrhodamine (only at the $\varepsilon$-amino group of HEWL), ${ }^{15}$ together with tetragonal HEWL crystals as the model substrate. For the second requirement, a transition process should be slow enough to allow sufficient time resolution for the observations, and hence, photobleaching ${ }^{16}$ of the fluorescent label has to be avoided during long-term observations. Therefore, we adopted pulsed discontinuous laser illumination, by which means we can decrease the total irradiation time of identical molecules and, hence, avoid photobleaching of the singlemolecule fluorescent dye.

With our technical solution, we demonstrate that it is possible to track the individual F-HEWL molecules during the transient processes of adsorption on HEWL crystals by thin-solutionlayer type single-molecule visualization, ${ }^{14}$ and we used a nonensemble analysis of the molecular movements.

\section{Experimental Section}

By optimizing the reaction conditions, the $\varepsilon$-amino group of the N-terminal of HEWL was specifically labeled with a fluorescent label, tetramethylrhodamin-5-isotiocyanate. ${ }^{15}$ The solution used for the adsorption measurements contained $1.01 \mathrm{mM}$ HEWL $(99.99 \%$ purity, Maruwa Food Industries, Inc., Japan), 0.1 nM F-HEWL, and $50 \mathrm{mM}$ sodium acetate $(\mathrm{pH} 4.5)$. The absence of aggregates of F-HEWL molecules in this solution was confirmed by nativepolyacrylamide gel electrophoresis. No deoxidation reagent, which is useful to prolong the fluorescence lifetime before photobleaching occurs, was used to avoid any deleterious effects on the HEWL crystals.

A total internal reflection fluorescence microscopy system using an objective was constructed on an inverted fluorescence microscope 


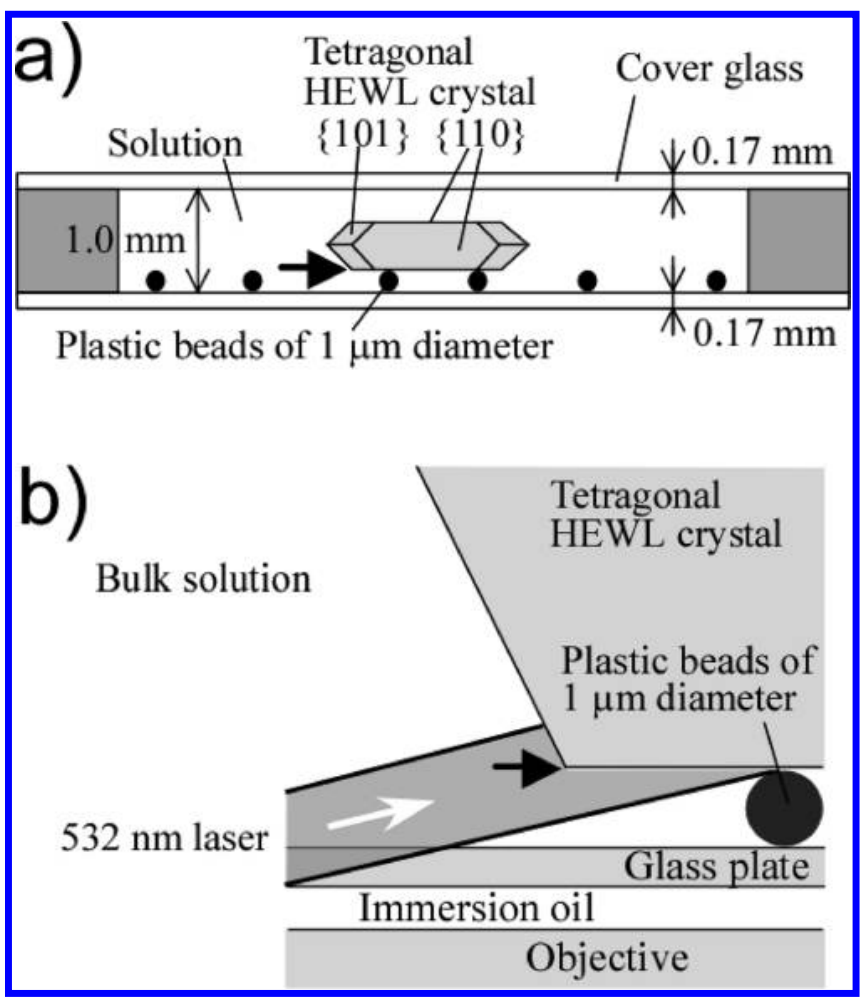

Figure 1. Schematic illustration of the experimental setup: (a) a sectional view of an observation chamber, and (b) an optical arrangement of the single-molecule visualization used in this study. The white arrow indicates the direction of the incident laser beam. The black arrow shows the observed interface between a solution and a tetragonal HEWL crystal.

(IX70, Olympus) equipped with a $60 \times$ oil-immersion objective (PlanApo 60× TIRFM3, Olympus). The F-HEWL molecules were illuminated with a $532 \mathrm{~nm}$ laser (fluence $0.11 \mathrm{~kW} / \mathrm{cm}^{2}$ ), and emission at $\geq 580 \mathrm{~nm}$ from F-HEWL was recorded using a dichroic mirror and an electron-multiplying charge-coupled device camera (EM-CCD; DV887; Andor Technology). As shown schematically in Figure 1a, plastic beads of $1 \mu \mathrm{m}$ diameter (FluoSpheres F8814, Molecular Probes, Inc.) were adsorbed on the lower glass plate of an observation chamber, and then a tetragonal HEWL crystal (several $100 \mu \mathrm{m}$ in height) and the sample solution were transferred into the chamber. The crystal was set on the $1 \mu \mathrm{m}$ beads to intentionally place a solution layer of $1 \mu \mathrm{m}$ thickness between the bottom surface of the HEWL crystal and the lower glass plate of the observation chamber. The solution-crystal interface, which was $1 \mu \mathrm{m}$ away from the bottom glass plate, was illuminated with a laser beam tilted almost parallel to the crystal surface (Figure 1b: several degrees from a horizontal direction) to avoid the increase in fluorescent background intensity. The focus of the objective was set at the interface marked by the bold arrow. Because of the diagonal illumination and small number of F-HEWL molecules inside a solution layer that is only $1 \mu \mathrm{m}$ thick, individual F-HEWL molecules were successfully observed in situ. The details of the experimental setup have been reported previously. ${ }^{14}$ The temperature inside the observation chamber was controlled at $20.0 \pm 0.1{ }^{\circ} \mathrm{C}$, a temperature at which the crystals and the solution were in equilibrium.

After the solution inside the chamber was replaced with the solution containing $0.1 \mathrm{nM} \mathrm{F-HEWL}$, the adsorption processes of F-HEWL on the bottom crystal surface were recorded. ${ }^{14}$ The frame rate of the EM-CCD camera was set at $0.1 \mathrm{~s}$, which is the lower limit for the detection of single F-HEWL molecules under our experimental conditions. To avoid photobleaching of F-HEWL, an electromagnetic shutter of the laser was sequentially opened and closed (as shown later in Figure $3 \mathrm{~d}$ schematically). Since the average lifetime for photobleaching was $\sim 40$ s under our experimental conditions, the observation was stopped within $31 \mathrm{~s}$ of total irradiation. Before and after the adsorption experiment, the surface morphology of the tetragonal HEWL crystal was

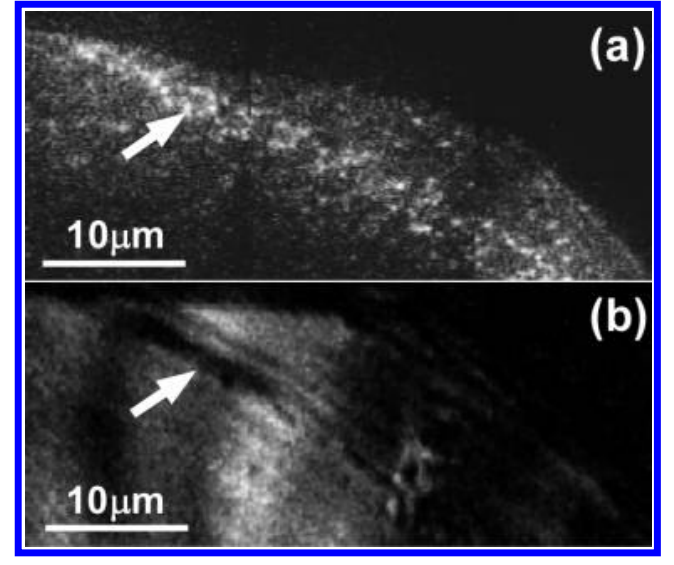

Figure 2. Comparison of two images taken by single-molecule visualization of a thin-solution-layer type ${ }^{14}$ (a) and by LCM-DIM ${ }^{17}$ (b). The same field of view on a $\{110\}$ face of a tetragonal HEWL crystal was observed by both methods 116 min after F-HEWL molecules were introduced into an observation chamber. Scale bars represent $10 \mu \mathrm{m}$. (a) One bright dot corresponds to one F-HEWL molecule adsorbed on the crystal surface. The image was composed of three frame images because of the limited field of view of the EM-CCD camera. In part $b$, an arrow shows the position where steps with a stronger contrast level can be observed, probably corresponding to bunched steps.

observed by laser confocal microscopy combined with differential interference contrast microscopy (LCM-DIM) ${ }^{17,18}$ to ensure that the crystal had been maintained in equilibrium during the adsorption experiment.

\section{Results and Discussion}

To investigate the dynamics of transition processes of crystal growth from a solution, one should know the adsorption sites of individual F-HEWL molecules on a crystal surface. Then we observed the same crystal surface by single-molecule visualization and LCM-DIM. Next, we observed the time-course of adsorption processes of individual F-HEWL molecules on a crystal surface and analyzed adsorbed F-HEWL molecules, using two kinds of time parameters: one is adsorption time $t_{\mathrm{ads}}$ (the elapsed time after F-HEWL molecules were introduced into the chamber), and the other is residence time $\tau$ (the time interval during which F-HEWL molecules were immobilized on a crystal surface).

We first investigated the adsorption sites of F-HEWL molecules on a $\{110\}$ face of the tetragonal HEWL crystal. Figure 2a shows a typical single-molecule image: one bright dot corresponds to one F-HEWL molecule adsorbed on the crystal surface. Figure $2 b$ represents the same field of view observed by LCM-DIM, ${ }^{17}$ by which molecular steps $(5.6 \mathrm{~nm}$ in height) on the HEWL crystals can be observed in situ. ${ }^{18}$ In Figure $2 b$, a white arrow shows the position where steps with a stronger contrast level, probably corresponding to bunched steps, were observed on the crystal surface. A comparison between images a and $b$ clearly demonstrates that F-HEWL molecules preferentially adsorbed on steps (not on a terrace). This result suggests that the F-HEWL molecules behaved as native HEWL molecules during the adsorption onto a HEWL crystal surface, ${ }^{18}$ unaffected by the presence of a low molecular weight fluorescent label $(<3 \%$ of the molecular weight of F-HEWL). Although we cannot completely ignore the effects of the fluorescent label on the adsorption process, we believe that the approach adopted in this study of F-HEWL was an effective way to explore the transient behavior of mobile 


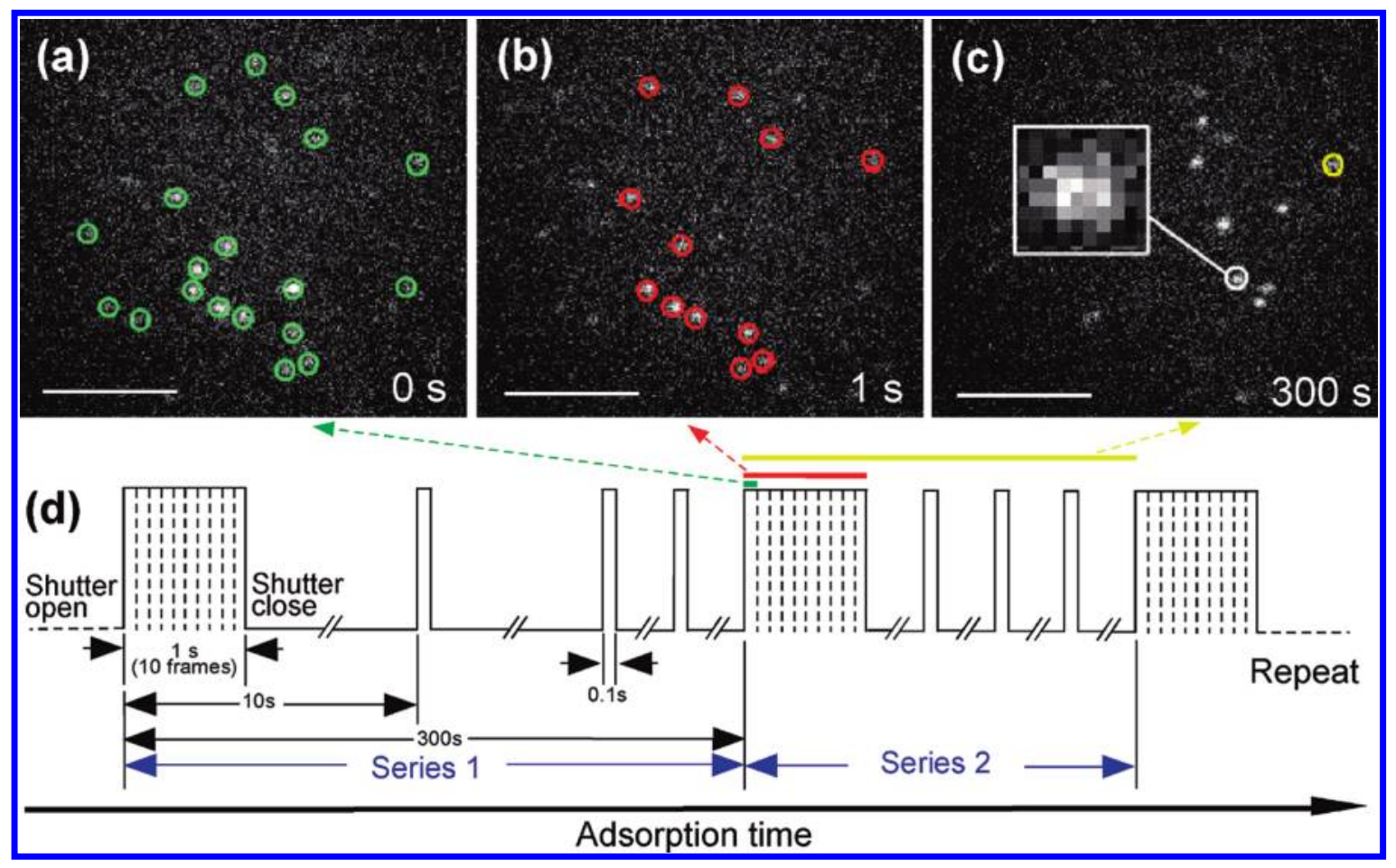

Figure 3. Nonensemble measurements of transient processes of adsorption on a HEWL crystal surface. (a-c) A typical time-course of F-HEWL molecules appearing on the crystal surface taken $61 \mathrm{~min}$ after the molecules were introduced into an observation chamber. Scale bars represent $5 \mu \mathrm{m}$. (a) Green circles show all the F-HEWL molecules visualized on the crystal surface. Red (b) and yellow (c) circles correspond to molecules whose positions were not changed for periods longer than 1 and $300 \mathrm{~s}$, respectively. (d) A time-sequence diagram of the pulsed discontinuous laser illumination.

solute molecules during adsorption at a solution-crystal interface, for the first time.

To distinguish the molecules immobilized on a crystal surface from those diffusing on a surface, we compared the positions of the molecules recorded in a series of time frames. Under our experimental conditions, one fluorescent spot exhibits a Gaussian distribution of several pixels to 6 pixels in diameter (the inset of Figure $3 \mathrm{c}$ ). ${ }^{14}$ When the center positions of spots in time frames were matched within one pixel size $(87 \mathrm{~nm})$, we judged that such a molecule did not change its position during that time interval. Parts a and $b$ of Figure 3 present examples of single-molecule images with a $1 \mathrm{~s}$ time interval. In Figure 3a, green circles correspond to all F-HEWL molecules visualized at the interface. In Figure $3 b$, red circles indicate the molecules whose positions were not changed during 1 s: i.e., the molecules immobilized on the crystal surface for at least $1 \mathrm{~s}$. Hereafter we define the molecules that were immobilized on the crystal surface as "adsorbed" molecules; in this case, the use of this word does not include molecules diffusing on the crystal surface.

In Figure 3, it is crucial to prove whether the same F-HEWL molecule was visualized at the same position in different frames: that is, whether we can reject the case in which one molecule desorbed and a second molecule adsorbed at the same location. There are two cases of adsorption to consider that would complicate this process: one is the direct adsorption of molecules from the bulk solution to the crystal surface, and the second is adsorption via surface diffusion of the molecules. We can reject the first case for the following reason. We previously found that the number density of the F-HEWL molecules observed at the interface is 3 orders of magnitude higher than that estimated from the concentration in a bulk solution, ${ }^{14}$ because of the significant condensation of the molecules by interactions with the crystal surface. In other words, the concentration of the F-HEWL molecules in the bulk solution is low enough that we can disregard direct adsorption from solution at the same location from which a previously adsorbed molecule has desorbed. We can also reject the second case of adsorption via surface diffusion. The average measured surface diffusion length is $0.11 \mu \mathrm{m},{ }^{14}$ which is significantly smaller than the average distance between the molecules observed in Figure 3a. Hence, a molecule diffusing on the surface would desorb before it reached the position of an adjacent molecule. Therefore, a bright spot that has not changed its position between two frames cannot be the result of one molecule desorbing and a second adsorbing at the same location, and it must correspond to a stationary molecule. Hereafter we define the time interval during which the molecule did not change its position on the crystal surface as the residence time $\tau$.

Figure $3 \mathrm{c}$ is an image taken $300 \mathrm{~s}$ after the image in Figure $3 \mathrm{a}$. The yellow circles indicate molecules that have not moved between the images in parts a and c of Figure 3 and, therefore, have a $\tau \geq 300 \mathrm{~s}$. Note that the number of molecules of $\tau \geq 300 \mathrm{~s}$ is significantly smaller than that of $\tau \geq 1 \mathrm{~s}$ (Figure $3 b$ ), indicating the significant desorption of the molecules during this period. Most of the molecules shown in Figure $3 c$ (not marked by circles) appeared on the crystal surface after Figure 3 a was taken.

We observed the movement of F-HEWL molecules using pulsed discontinuous laser illumination according to the procedure shown in Figure 3d. At a given elapsed time after the introduction of F-HEWL molecules into the chamber, $t_{\mathrm{ads}}$, we determined the number densities $N$ of the molecules whose positions had not changed during a series of residence times $\tau$. Hence, the number density $N\left(t_{\mathrm{ads}}, \geq \tau\right)$ does not include those molecules that were newly adsorbed during a time $\tau$ after $t_{\text {ads. }}$. 

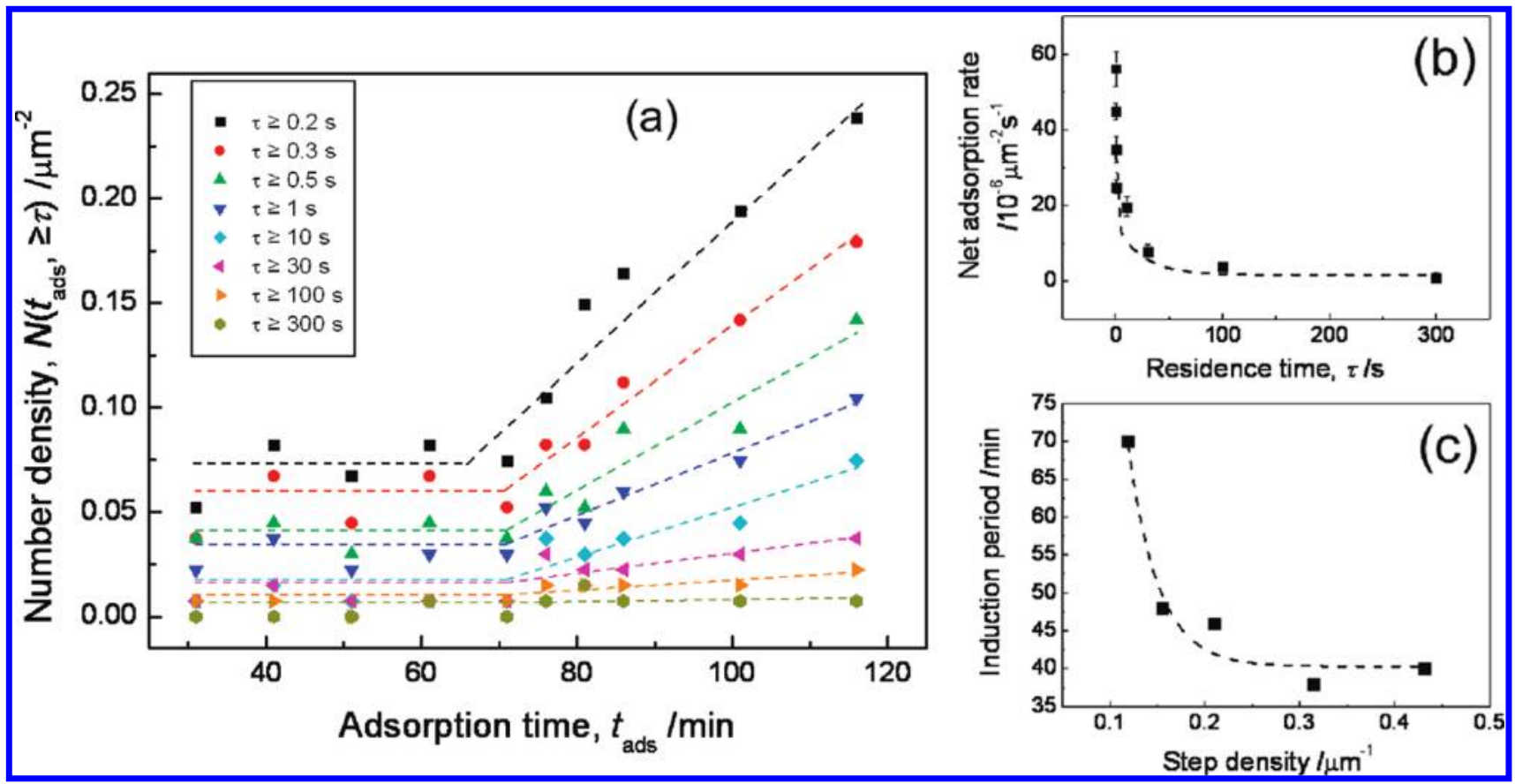

Figure 4. Dynamics of transient processes during adsorption. (a) Changes in number density $N\left(t_{\text {ads }}, \geq \tau\right)$ of F-HEWL molecules whose positions were not changed, within an accuracy of one pixel size, for longer than a residence time $\tau$, as a function of an adsorption time $t_{\text {ads. }}$. The residence time $\tau$ was varied from 0.2 to $300 \mathrm{~s}$. The total irradiation time of the crystal surface with the pulsed discontinuous laser illumination was $31 \mathrm{~s}$, and the step density of the observed crystal surface was $0.12 \mu \mathrm{m}^{-1}$. (b) Effects of the residence time $\tau$ on the net adsorption rate of F-HEWL molecules, corresponding to the slope of the dashed lines in part a after the induction period ( $~ 70$ min). Error bars were evaluated from the standard deviation of the linear fits. (c) Effects of the step density on the induction period of F-HEWL molecules during adsorption.

When $\tau$ is small, $N\left(t_{\text {ads }}, \geq \tau\right)$ includes the contribution of surface diffusion of F-HEWL molecules at the solution-crystal interface. We previously found that the average two-dimensional (2D) diffusion coefficient of F-HEWL molecules at the solution-crystal interface was $(6.9 \pm 1.2) \times 10^{-15} \mathrm{~m}^{2} / \mathrm{s}^{14}$ Hence, during a minimum residence time of $0.2 \mathrm{~s}$, the molecules at the interface diffuse $74 \mathrm{~nm}$ in 2D. Since the accuracy of the position determination in this study was $87 \mathrm{~nm}$ (the size of one pixel $), N\left(t_{\mathrm{ads}}, \geq 0.2 \mathrm{~s}\right)$ includes both the adsorbed molecules and those diffused at the interface. With increasing $\tau$, adsorbed molecules dominate $N\left(t_{\mathrm{ads}}, \geq \tau\right)$. Adsorbed molecules are a majority of the molecules observed at the interface for $\tau>0.47 \mathrm{~s}$, which corresponds to a $\tau$ with the maximum population of diffusing molecules. ${ }^{14}$

We obtained systematic changes in $N\left(t_{\mathrm{ads}}, \geq \tau\right)$ for various $\tau$, as a function of $t_{\text {ads }}$ (Figure 4a). We found an induction period ( $\sim 70 \mathrm{~min}$ ) before the sudden increase of $N\left(t_{\mathrm{ads}}, \geq \tau\right)$. After the induction period, $N\left(t_{\mathrm{ads}}, \geq \tau\right)$ increased linearly with the adsorption time: the dashed lines after the induction period in Figure 4a show the results of linear fits. Our results are different from those of previous traditional adsorption studies, ${ }^{19-21}$ in which no induction period was observed ( $N$ started to increase from $t_{\mathrm{ads}}=0$ ). The existence of the induction period indicates that the adsorption did not proceed through a singlestep adsorption process. If a single-step adsorption process is the case, the probability that an individual molecule overcomes the activation energy barrier to the process is proportional to the number of attempts, i.e. to the elapsed time, $t_{\text {ads }}$. Therefore, the results shown in Figure 4a suggest that the adsorption proceeded through a series of successive multistep processes. We assume that dehydration of protein molecules might be a likely cause for the series of successive multistep processes occurring during adsorption, although we do not have any experimental evidence for such hypotheses.
Furthermore, Figure 4a shows that, with increasing $\tau$ at the same $t_{\mathrm{ads}}, N\left(t_{\mathrm{ads}}, \geq \tau\right)$ becomes significantly smaller. This result clearly demonstrates that stronger adsorption with a longer $\tau$ was less likely than weaker adsorption with a shorter $\tau$, implying that the adsorption of molecules onto the crystal surface became gradually stronger over a number of trials. The gradual immobilization of solute molecules on a crystal surface corresponds to the gradual change in an order parameter during a transition from solute to solid states.

From the linear fits of $N\left(t_{\text {ads }}, \geq \tau\right)$ vs $t_{\text {ads }}$ plots after the induction period, we determined the net adsorption rates shown in Figure $4 \mathrm{~b}$ as a function of $\tau$. With increasing $\tau$, the net adsorption rate drastically decreased. This result supports the idea of adsorption becoming more difficult with increasing $\tau$. Figure $4 \mathrm{~b}$ strongly suggests that the crystal growth at a solution-crystal interface does not proceed abruptly: molecules first adsorb on a crystal surface weakly, and then the residence time of molecules gradually increases, and finally molecules become a part of the solid phase.

Beyond a residence time of $100 \mathrm{~s}$, the net adsorption rate appeared to be almost zero (Figure 4b). In this study, although the HEWL solution and the crystal were in equilibrium (steps on the crystal surface did not grow laterally), F-HEWL molecules were initially included only in the HEWL solution. Hence, the adsorption of F-HEWL molecules onto the crystal surface proceeded until $N\left(t_{\mathrm{ads}}, \geq \tau\right)$ reached a certain equilibrium value. Therefore, with increasing $\tau$, the net adsorption rate could finally reach a positive constant value, which corresponds to the permanent adsorption rate by which the F-HEWL molecules finally integrated into the crystal surface and became a part of the crystal. To measure the permanent adsorption rate, measurements (1) at much longer $t_{\text {ads }}$ or (2) using a solution of much higher F-HEWL concentration have to be performed. However, the measurement (1) is technically very difficult 
because of the photobleaching of F-HEWL caused by the laser illumination time being longer than the lifetime of F-HEWL. The measurement (2) is also technically very difficult, since the high density of F-HEWL molecules appearing on the crystal surface disabled us from visualizing individual F-HEWL molecules.

We also found that the induction period strongly depended on the step density of the crystal surface (total step length for a unit area), i.e. the density of adsorption sites. Figure $4 \mathrm{c}$ shows changes in the induction period as a function of the step density. The induction period monotonically decreased with increasing step density. This result clearly demonstrates that the adsorption sites participated synergistically in the successive multistep adsorption processes and strongly influenced them. Again, we suppose that changes in chemical conditions at the adsorption sites (steps), such as hydration, are responsible for triggering adsorption.

In this preliminary study, we demonstrated the novel picture of immobilization processes that was not incorporated in the traditional adsorption theory. However, there are still many factors that also have to be taken into account. One such important factor is supersaturation, which drives crystallization. In the thin-layer-type optical arrangement (Figure 1), when we perform similar experiments using supersaturated HEWL solutions, we cannot avoid the depletion of solute inside the thin solution layer of $1 \mu \mathrm{m}$ thickness. Therefore, a similar single-molecule observation has to be carried out at the interface between a bulk supersaturated solution and a top (free) crystal surface. To avoid optical aberration caused by a protein crystal of several $100 \mu \mathrm{m}$ thickness, ${ }^{14}$ we will perform a similar experiment using a protein crystal with a thickness smaller than several $10 \mu \mathrm{m}$.

In this study, we focused on the F-HEWL molecules immobilized at the step edges. However, their dynamic behavior also includes important insight into crystal growth from a solution. To understand the entire processes of solution growth, we are also planning to analyze diffusion at step ledges in the future, in addition to immobilization at step edges (this study) and diffusion on a terrace. ${ }^{14}$

We emphasize that no experimental work has yet been reported on the transient behavior of individual molecules during adsorption/desorption processes. To find the induction period, the adsorption process had to be slow enough so that true early stages could be observed. Hence, ordinary means, such as a quartz crystal microbalance, are not sensitive enough. ${ }^{22}$ In addition, the observation has to be a nonensemble measurement to find the amount of the adsorbed molecules as a function of the residence time. However, the observation also has to be noninvasive. Therefore, scanning probe microscopy, a popular technique for nonensemble observations of a solid surface at the molecular level, is not appropriate because of significant disturbance of mobile solute molecules induced by the scan of a cantilever. The use of single-molecule visualization at the solution-crystal interface using discontinuous pulsed illumination enabled us to discover such processes.

\section{Conclusion}

In the previous studies of adsorption kinetics, no induction period has been detected and the adsorption rate was thought to be constant during the initial stage of an adsorption process. ${ }^{23}$ However, in this study the nonensemble analysis revealed a nontraditional picture of the initial transient process of adsorption: the adsorption of F-HEWL molecules on the HEWL crystal exhibited an induction period for adsorption, indicating the existence of successive multistep processes, by which the molecules were gradually immobilized on the adsorption sites. Since hydrogen bonding, which is the main intermolecular interaction between protein molecules, is a common interaction in a wide variety of systems, the mechanism envisaged in this study could apply directly to a wide variety of systems, in particular to transient processes of crystal growth at an interface between an aqueous solution and hydrophilic solid, where electrostatic interactions play a key role.

Acknowledgment. The authors thank H. Higuchi (The University of Tokyo) for valuable discussions, and they acknowledge grants from the National Basic Research Program of China (973 Program) (G.D.: No. 2011CB710901), the Knowledge Innovation Program of the Chinese Academy of Sciences (G.D.: No. KJCX2-YW-L08), and the National Nature Science Foundation of China (G.D.: No. 20603043), and Grants-in-Aid (G.S.: Nos. 17034007 and 18360003) from the Scientific Research of the Ministry of Education, Science and Culture, Japan. One of the authors (G.D.) acknowledges a grant from Tohoku University as a visiting professor. This work was carried out as a part of "Project Research B" at the Center for Interdisciplinary Research, Tohoku University, as well as the "Ground-based Research Announcement for Space Utilization" of the Japan Space Forum.

\section{References}

(1) Wirth, M. J.; Swinton, D. J.; Ludes, M. D. J. Phvs. Chem. B 2003, 107, 6258-6268.

(2) Kang, S. H.; Yeung, E. S. Anal. Chem. 2002, 74, 6334-6339.

(3) Xu, X.-H. N.; Yeung, E. S. Science 1998, 281, 1650-1653.

(4) Funatsu, T.; Harada, Y.; Tokunaga, M.; Saito, K.; Yanagida, T. Nature 1995, 374, 555-559.

(5) Sako, Y.; Uyemura, T. Cell Struct. Funct. 2002, 27, 357-365.

(6) Cognet, L.; Coussen, F.; Choquet, D.; Lounis, B. C. R. Phvs. 2002, $3,645-656$

(7) Wazawa, T.; Ueda, M. Adv. Biochem. Eng. Biotechnol. 2005, 95, $77-106$.

(8) Kellermayer, M. S. Z. Phvsiol. Meas. 2005, 26, R119-R153.

(9) Andreas, W. S.; Ulla, M. E.; Thomas, H. C.; Hjalmar, B. Langmuir 2007, 23, 8352-8356.

(10) Xu, X. H.; Yeung, E. S. Science 1997, 275, 1106-1109.

(11) Kirstein, J.; Platschek, B.; Jung, C.; Brown, R.; Bein, T.; Bräuchle, C. Nat. Mater. 2007, 6, 303-310.

(12) Werley, C. A.; Moerner, W. E. J. Phvs. Chem. B 2006, 110, 18939 18944.

(13) Maarten, B. J. R.; Bert, F. S.; Hiroshi, U.; Frans, C. D. S.; Pierre, A. J.; Dirk, E. D. V.; Johan, H. Nature 2006, 439, 572-575.

(14) Sazaki, G.; Okada, M.; Matsui, T.; Watanabe, T.; Higuchi, H.; Tsukamoto, K.; Nakajima, K. Crvst. Growth Des. 2008, 8, 2024 2031.

(15) Matsui, T.; Sazaki, G.; Hondoh, H.; Matsuura, Y.; Nakada, T.; Nakajima, K. J. Crvst. Growth 2006, 293, 415-422.

(16) Lippitz, M.; Kulzer, F.; Orrit, M. ChemPhvsChem 2005, 6, 770-789.

(17) Sazaki, G.; Matsui, T.; Tsukamoto, K.; Usami, N.; Ujihara, T.; Fujiwara, K.; Nakajima, K. J. Crvst. Growth 2004, 262, 536-542.

(18) Van Driessche, E. S.; Sazaki, G.; Otálora, F.; González-Rico, F. M.; Dold, P.; Tsukamoto, K.; Nakajima, K. Crvst. Growth Des. 2007, 7, 1980-1987.

(19) Guesmi, H.; Lapena, L.; Ranguis, A.; Muller, P.; Treglia, G. Phvs. Rev. Lett. 2005, 94, 076101.

(20) Puskas, J. E.; Dahman, Y.; Margaritis, A. Biomacromolecules 2004, $5,1412-1421$

(21) Nagaoka, H.; Imae, T. J. Colloid Interface Sci. 2003, 264, 335-342.

(22) The minimum amount of adsorbate molecules necessary for the quartz crystal microbalance is $0.1 \mathrm{ng}$, which corresponds to $9 \times 10^{17}$ HEWL molecules; this value is much larger than the number of the molecules observed on the crystal surface in this study. Kenneth, A. M. Biomacromolecules 2003, 4, 1099-1120.

(23) Islam, S. A.; Weaver, S. L. Proteins 1990, 8, 1-5. 